\title{
Optimal control model of immunotherapy for autoimmune diseases
}

\author{
M. Fernanda Costa ${ }^{1}$, M. Piedade $\operatorname{Ramos}^{1}$, Carolina Ribeiro ${ }^{1}$, and Ana Soares ${ }^{1}$ \\ ${ }^{1}$ University of Minho
}

August 21, 2020

\begin{abstract}
In this work, we develop a new mathematical model to evaluate the impact of drug therapies on autoimmunity disease. We describe the immune system interactions at the cellular level, using the kinetic theory approach, by considering self-antigen presenting cells, self-reactive $\mathrm{T}$ cells, immunosuppressive cells and Interleukin-2 (IL-2) cytokines. The drug therapy consists of an intake of Interleukin-2 cytokines which boosts the effect of immunosuppressive cells on the autoimmune reaction. We also derive the macroscopic model relative to the kinetic system and study the wellposedness of the Cauchy problem for the corresponding system of equations. We formulate an optimal control problem relative to the model so that the quantity of both the self-reactive $\mathrm{T}$ cells that are produced in the body and the Interleukin- 2 cytokines that are administrated is simultaneously minimized. Moreover, we perform some numerical tests in view of investigating optimal treatment strategies and the results reveal that the optimal control approach provides good-quality approximate solutions and shows to be a valuable procedure in identifying optimal treatment strategies.
\end{abstract}

\section{Hosted file}

SoaresRibeiroRamosCosta.pdf available at https://authorea.com/users/352767/articles/476898optimal-control-model-of-immunotherapy-for-autoimmune-diseases

\section{Hosted file}

SoaresRibeiroRamosCosta.tex available at https://authorea.com/users/352767/articles/476898optimal-control-model-of-immunotherapy-for-autoimmune-diseases 
figures/Plot-SAPC/Plot-SAPC-eps-converted-to.pdf 
figures/Plot-SRTC/Plot-SRTC-eps-converted-to.pdf 
figures/Plot-ISC/Plot-ISC-eps-converted-to.pdf 
figures/Fig-u(t)/Fig-u(t)-eps-converted-to.pdf 
figures/Fig-A(t)/Fig-A(t)-eps-converted-to.pdf 
figures/Fig-R(t)/Fig-R(t)-eps-converted-to.pdf 
figures/Fig-S(t)/Fig-S(t)-eps-converted-to.pdf 
figures/f2-without-Control-cons/f2-without-Control-cons-eps-converted-to.pdf 
figures/f2-withControl-c-null/f2-withControl-c-null-eps-converted-to.pdf 
figures/f2-withControl-c-non-null/f2-withControl-c-non-null-eps-converted-to.pdf 
figures/f2-withControl-c23-0-02/f2-withControl-c23-0-02-eps-converted-to.pdf 
figures/f2-withControl-c23-0-2/f2-withControl-c23-0-2-eps-converted-to.pdf 
figures/f3-withControl-c34-0-01/f3-withControl-c34-0-01-eps-converted-to.pdf 
figures/f3-withControl-c34-0-2/f3-withControl-c34-0-2-eps-converted-to.pdf 
figures/f3-withControl-c34-2/f3-withControl-c34-2-eps-converted-to.pdf 